DOI: https://doi.org/10.14311/TPFM.2022.001

\title{
STABILITY OF NONISOTHERMAL POISEUILLE FLOW IN A FLUID OVERLY- ING A HIGHLY POROUS DOMAIN
}

\author{
Anjali $^{1}$, P. Bera ${ }^{1}$ \\ ${ }^{1}$ Department of Mathematics, Indian Institute of Technology Roorkee, Roorkee-247667, \\ India
}

\begin{abstract}
The present article examines the stability of nonisothermal Poiseuille flow in a highly permeable porous domain underlying a fluid domain. A two domain approach with the Newtonian fluid's flow being governed by Navier-Stokes equations in the fluid domain and Darcy- Brinkman model in the porous domain is adopted. The impact of depth ratio (ratio of fluid domain's depth to porous domain's depth), Darcy number, Reynolds number and Prandtl number are analyzed with the aid of linear stability analysis. The neutral stability curves characterize unimodal (either porous or fluid) or bimodal (both porous and fluid) behavior depending on the parametric variation. A decrease in depth ratio and increase in Darcy number, Reynolds number and Prandtl number instigates the instability in porous domain. A qualitative comparison with the existing results on Darcy's law is also presented.
\end{abstract}

Keywords: mixed convection, fluid-porous system, buoyancy-driven instability.

\section{Introduction}

The phenomena of convection in fluid overlying porous domain finds large scale application in geophysical viz. water flow beneath earth's surface [1], oil flow in underground reservoirs [2], industrial viz. cooling of electronic components [3], flow in fuel cells [4], etc. Thermal convection in fluid overlying porous domain, wherein the porous media was modelled by Darcy's law was studied by Sun [5], Chen \& Chen [6], Chang [7], Chang et al. [8] and Yin et al. [9]. However, while dealing with the highly porous domain, Darcy-Brinkman model is recommended to model the porous media flow [10]. Desaive \& Lebon [11] were the first to study the natural convection in superposed systems using Brinkman model. They carried out linear stability analysis to examine Marangoni effect and also made a comparison with the existing Darcy law's results. They observed qualitatively same result for Brinkman and Darcy's law. The instability thresholds for linear and nonlinear stability in natural convection in fluid overlying highly porous material was investigated by Hill \& Straughan [12]. Their results concluded good agreement between linear and nonlinear stability bounds for foametal. Isothermal studies on fluid overlying highly porous media can be found in works of Liu et al. [13] and Hill \& Straughan [14]. A question that pops up is whether one can find a good accord between Darcy's and Brinkman's model for mixed convection? Also, how the parametric study for mixed convection in superposed system alters in the presence of highly porous surfaces is an interesting direction to explore. To seek answers to these, the present article deals with linear stability analysis of thermal convection subjected to plane Poiseuille flow in fluid overlying highly porous domain.

\section{Formulation of the Problem}

\subsection{The Physical Model}

The present system (schematic diagram shown in Figure 1) consists of a horizontal porous domain underlying a fluid domain of depth $d_{m}$ and $d$, respectively. The flow of the incompressible Newtonion fluid is regulated by Navier-Stokes equations in the fluid domain whereas in the porous domain, the flow is governed by Darcy Brinkman's Law. The Cartesian system with $x$ and $x_{m}\left(z\right.$ and $\left.z_{m}\right)$ denotes the mean flow direction (the vertical direction) in the fluid and porous domain, respectively. The introduction of Poiseuille flow is via the application of constant pressure gradient and convection is by means of constant temperature at the upper $\left(T_{U}\right)$ and lower layer $\left(T_{L}\right)$ of the system, with $\left(T_{L}>T_{U}\right)$. The governing equations, in 


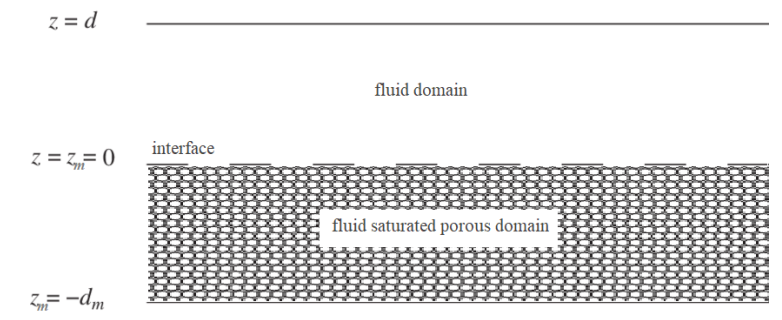

Figure 1: Schematic diagram of the considered system

dimensional form, for mass, momentum and energy conservation in fluid domain are

$$
\begin{aligned}
& \frac{\partial u}{\partial x}+\frac{\partial w}{\partial z}=0, \\
& \frac{\partial u}{\partial t}+u \frac{\partial u}{\partial x}+w \frac{\partial u}{\partial z}=-\frac{1}{\rho_{o}} \frac{\partial p}{\partial x}+\nu \Delta u, \\
& \frac{\partial w}{\partial t}+u \frac{\partial w}{\partial x}+w \frac{\partial w}{\partial z}=-\frac{1}{\rho_{o}} \frac{\partial p}{\partial z}+\nu \Delta w-g\left[1-\alpha_{T}\left(T-T_{o}\right)\right], \\
& \frac{\partial T}{\partial t}+u \frac{\partial T}{\partial x}+w \frac{\partial T}{\partial z}=\alpha \Delta T,
\end{aligned}
$$

where $u$ and $w$ denotes the $x$ and $z$ components of velocity for fluid domain respectively, $p, \nu, T, \rho_{o}, \Delta, g$, $\alpha_{T}$ and $\alpha$ denotes pressure, kinematic viscosity, temperature, density at reference temperature $T_{o}$, Laplacian operator in two-dimension, gravity, coefficient of thermal expansion and thermal diffusivity respectively.

The dimensional equations in the porous domain for mass, momentum and energy conservation are

$$
\begin{aligned}
& \frac{\partial u_{m}}{\partial x_{m}}+\frac{\partial w_{m}}{\partial z_{m}}=0 \\
& \frac{1}{\chi} \frac{\partial u_{m}}{\partial t_{m}}=-\frac{1}{\rho_{o}} \frac{\partial p_{m}}{\partial x_{m}}-\frac{\nu}{K} u_{m}+\frac{\nu}{\chi} \Delta u_{m} \\
& \frac{1}{\chi} \frac{\partial w_{m}}{\partial t_{m}}=-\frac{1}{\rho_{o}} \frac{\partial p_{m}}{\partial z_{m}}-\frac{\nu}{K} w_{m}+\frac{\nu}{\chi} \Delta w_{m}-g\left[1-\alpha_{T}\left(T_{m}-T_{o}\right)\right] \\
& G_{m} \frac{\partial T_{m}}{\partial t_{m}}+u_{m} \frac{\partial T_{m}}{\partial x_{m}}+w_{m} \frac{\partial T_{m}}{\partial z_{m}}=\alpha_{m} \Delta T_{m},
\end{aligned}
$$

where the quantities with subscript $m$ represent the respective quantities in porous domain and $\chi$ denote the porosity. Also, $G_{m}=\left(\rho_{o} c_{p}\right)^{*} / \rho_{o} c_{p}$ and $\alpha_{m}=\kappa^{*} / \rho_{o} c_{p}$ with the relationship

$$
X^{*}=\chi X_{f}+(1-\chi) X_{m},
$$

where $X$ can be replaced by $\rho_{o} c_{p}$ or $\kappa, c_{p}$ the specific heat of the fluid accordingly. The subscripts $f$ and $m$ represents respectively, the fluid and the porous quantities.

The boundary conditions for the system are as follows:

At the upper surface of fluid domain i.e. $z=d$,

$$
u=w=0, T=T_{U}
$$

At the bottom surface of porous domain i.e. $z_{m}=-d_{m}$,

$$
u_{m}=0, w_{m}=0, T_{m}=T_{L} .
$$

At the fluid-porous interface i.e. $z=z_{m}=0$, the velocity, temperature and stress' continuity [15] gives rise to

$$
u=u_{m}, \quad w=w_{m}, \quad T=T_{m}, \quad \alpha \frac{\partial T}{\partial z}=\alpha_{m} \frac{\partial T_{m}}{\partial z_{m}}, \quad p-2 \mu \frac{\partial w}{\partial z}=p_{m}-2 \frac{\mu}{\chi} \frac{\partial w_{m}}{\partial z_{m}} \text { and } \frac{1}{\chi} \frac{\partial u_{m}}{\partial z_{m}}=\frac{\partial u}{\partial z},
$$

where $\mu$ denotes the dynamic viscosity of the fluid. 


\subsection{The basic flow}

A steady, fully developed and one dimensional basic flow solution for the Poiseuille flow is given by

$$
\begin{aligned}
& \bar{u}=\frac{A_{1}}{2} z^{2}+A_{2} z+A_{3}, \quad \bar{w}=0, \\
& \bar{T}(z)=\frac{\left(T_{U}-T_{o}\right)}{d} z+T_{o},
\end{aligned}
$$

in the fluid domain, where $T_{o}$ is the temperature at the fluid-porous interface. The basic flow solution in the porous domain is

$$
\begin{aligned}
& \bar{u}_{m}\left(z_{m}\right)=C_{1} e^{\left(\sqrt{\chi / K} z_{m}\right)}+C_{2} e^{\left(-\sqrt{\chi / K} z_{m}\right)}-K A_{1}, \quad \bar{w}_{m}=0, \\
& \bar{T}_{m}\left(z_{m}\right)=\frac{\left(T_{o}-T_{L}\right)}{d_{m}} z_{m}+T_{o} .
\end{aligned}
$$

In equations (12) and (14), the different constants are

$$
\begin{gathered}
A_{1}=\frac{1}{\mu} \frac{\partial p}{\partial x}, A_{2}=\frac{-\left(4 A_{1} \chi e^{\left(\sqrt{\chi / K} d_{m}\right)}-2 A_{1} \chi-2 A_{1} \chi e^{2\left(\sqrt{\chi / K} d_{m}\right)}+A_{1} \chi d^{2} / K+A_{1} \chi d^{2} e^{2\left(\sqrt{\chi / K} d_{m}\right)} / K\right)}{\left(2 \sqrt{\chi / K}\left(d \sqrt{\chi / K}-\chi+\chi e^{2\left(\sqrt{\chi / K} d_{m}\right)}+d e^{2\left(\sqrt{\chi / K} d_{m}\right)} \sqrt{\chi / K}\right)\right)}, \\
A_{3}=\frac{-\left(2 A_{1} \chi d-A_{1} \chi d^{2} \sqrt{\chi / K}-4 A_{1} \chi d e^{\left(\sqrt{\chi / K} d_{m}\right)}+2 A_{1} \chi d e^{2\left(\sqrt{\chi / K} d_{m}\right)}+A_{1} \chi d^{2} e^{2\left(\sqrt{\chi / K} d_{m}\right)} \sqrt{\chi / K}\right)}{\left(2 \sqrt{\chi / K}\left(d \sqrt{\chi / K}-\chi+\chi e^{2\left(\sqrt{\chi / K} d_{m}\right)}+d e^{2\left(\sqrt{\chi / K} d_{m}\right)} \sqrt{\chi / K}\right)\right)}, \\
C_{1}=\frac{-\left(A_{1} K\left(2 \chi e^{\left(\sqrt{\chi / K} d_{m}\right)}-2 \chi e^{2\left(\sqrt{\chi / K} d_{m}\right)}-2 d e^{\left(\sqrt{\chi / K} d_{m}\right)} \sqrt{\chi / K}+\chi d^{2} e^{2\left(\sqrt{\chi / K} d_{m}\right)} / K\right)\right)}{\left(2\left(d \sqrt{\chi / K}-\chi+\chi e^{2\left(\sqrt{\chi / K} d_{m}\right)}+d e^{2\left(\sqrt{\chi / K} d_{m}\right)} \sqrt{\chi / K}\right)\right)} \\
C_{2}=\frac{A_{1} K\left(2 \chi e^{(\sqrt{\chi / K}} d_{m}\right)}{\left(2\left(d \sqrt{\chi / K}-\chi+\chi e^{2\left(\sqrt{\chi / K} d_{m}\right)}+d e^{2\left(\sqrt{\chi / K} d_{m}\right)} \sqrt{\chi / K}\right)\right)} .
\end{gathered}
$$

\subsection{Linearized Perturbed Equations}

The governing equations are non dimensionalized using $d, V, d / V, \mu V / d$ and $\left(T_{o}-T_{U}\right) \nu / \alpha$ as respective scales for length, velocity, time, pressure and temperature in the fluid domain, where $V$ is the maximum of $\bar{u}(z)$ and using $d_{m}, V_{m}, d_{m} / V_{m}, \mu V_{m} / d_{m}$ and $\left(T_{L}-T_{o}\right) \nu / \alpha_{m}$ as analogous scales in the porous domain, where $V_{m}=\bar{u}_{m}(0)$. The same scale for velocities are used to non dimensionalize the basic velocities in respective domains. To investigate the linear stability of basic flow, the flow variables are split into a basic flow variable and an infinitesimal disturbance, as

$$
(u, w, T, p)=(\bar{U}(z), 0, \bar{T}(z), P(x))+\left(u^{\prime}, w^{\prime}, T^{\prime}, p^{\prime}\right)
$$

for the fluid domain and

$$
\left(u_{m}, w_{m}, T_{m}, p_{m}\right)=\left(\overline{U_{m}}\left(z_{m}\right), 0, \overline{T_{m}}\left(z_{m}\right), P_{m}\left(x_{m}\right)\right)+\left(u_{m}^{\prime}, w_{m}^{\prime}, T_{m}^{\prime}, p_{m}^{\prime}\right)
$$

for the porous domain with $\bar{U}$ and $\bar{U}_{m}$ denoting the non dimensional basic velocities in the fluid and porous domain respectively. After superimposing the infinitesimal disturbances, eliminating the pressure terms and applying normal mode [16] as

$$
\begin{aligned}
& (w, T)=[W(z), \theta(z)] \exp [-\iota \sigma t+\iota a x] \\
& \left(w_{m}, T_{m}\right)=\left[W_{m}\left(z_{m}\right), \theta_{m}\left(z_{m}\right)\right] \exp \left[-\iota \sigma_{m} t_{m}+\iota a_{m} x_{m}\right]
\end{aligned}
$$


(with dropping of prime superscript for perturbed state notationally) gives the linearized perturbed equations for fluid domain as

$$
\begin{aligned}
& \left(D^{2}-a^{2}-\iota a \operatorname{Re} \bar{U}\right)\left(D^{2}-a^{2}\right) W+\iota a \operatorname{Re} \frac{d^{2} \bar{U}}{d z^{2}} W-\frac{R a}{R e} a^{2} \theta=-\iota \sigma \operatorname{Re}\left(D^{2}-a^{2}\right) W, \\
& \left(D^{2}-a^{2}\right) \theta-\iota a \operatorname{Re} \operatorname{Pr} \bar{U} \theta+\operatorname{Re} W=-\iota \sigma \operatorname{Pr} \operatorname{Re} \theta,
\end{aligned}
$$

and for the porous domain as

$$
\begin{aligned}
& -\frac{\left(D_{m}^{2}-a_{m}^{2}\right)^{2} W_{m}}{\chi}+\frac{\left(D_{m}^{2}-a_{m}^{2}\right) W_{m}}{\delta^{2}}+\frac{R a_{m}}{\delta^{2} R e_{m}} a_{m}^{2} \theta_{m}=\iota \frac{\sigma_{m} R e_{m}}{\chi}\left(D_{m}^{2}-a_{m}^{2}\right) W_{m}, \\
& \left(D_{m}^{2}-a_{m}^{2}\right) \theta_{m}-\iota a_{m} \bar{U}_{m} P r_{m} \operatorname{Re}_{m} \theta_{m}+R e_{m} W_{m}=-\iota P r_{m} R e_{m} G_{m} \sigma_{m} \theta_{m},
\end{aligned}
$$

where

$$
D=\frac{d}{d z}, \quad D_{m}=\frac{d}{d z_{m}}, \quad a=\hat{d} a_{m} \text { and } \sigma=\frac{\hat{d}^{2} R e_{m}}{R e} \sigma_{m}
$$

Here, $a$ and $a_{m}$ denotes the streamwise wavenumber in the fluid and porous domain respectively, $\sigma$ and $\sigma_{m}$ denotes the complex wavespeed in the fluid and porous domain respectively. Similarly, the boundary conditions become,

At $z=1, \quad W=D W=\theta=0$.

At $z_{m}=-1, \quad W_{m}=D_{m} W_{m}=\theta_{m}=0$.

At $z=z_{m}=0, \quad \operatorname{Re} W=\hat{d} R e_{m} W_{m}, \quad \operatorname{Re} D W=\hat{d}^{2} R e_{m} D_{m} W_{m}$,

$$
\begin{gathered}
\hat{d} \theta=\epsilon^{2} \theta_{m}, \quad D \theta=\epsilon D_{m} \theta_{m}, \quad \operatorname{Re} D^{2} W=\frac{\hat{d}^{3}}{\chi} \operatorname{Re}_{m} D_{m}^{2} W_{m}, \\
D^{3} W-3 a^{2} D W-\iota a \operatorname{Re} \bar{U} D W+\iota a \operatorname{Re} \frac{d \bar{U}}{d z} W+\frac{\hat{d}^{4} R e_{m} D_{m} W_{m}}{\delta^{2} R e}+\frac{2 a^{2} \hat{d}^{2} R e_{m} D_{m} W_{m}}{\chi R e} \\
-\frac{\hat{d}^{4} R e_{m} D_{m}^{3} W_{m}}{\chi R e}+\frac{\hat{d}^{4} a_{m}^{2} \operatorname{Re} e_{m} D_{m} W_{m}}{\chi R e}=-\iota \sigma R e D W+\iota \sigma_{m} \frac{\hat{d}^{4} R e_{m}^{2} D_{m} W_{m}}{\chi R e}
\end{gathered}
$$

where

$$
R e=\frac{V d}{\nu}, \quad R a=\frac{g \alpha_{T}\left(T_{o}-T_{U}\right) d^{3}}{\nu \alpha} \text { and } \operatorname{Pr}=\frac{\nu}{\alpha}
$$

denotes the Reynolds number, Rayleigh number and Prandtl number, respectively and

$$
R e_{m}=\frac{V_{m} d_{m}}{\nu}, R a_{m}=\frac{g \alpha_{T}\left(T_{L}-T_{o}\right) d_{m} K}{\nu \alpha_{m}} \text { and } P r_{m}=\frac{\nu}{\alpha_{m}}
$$

denotes the porous Reynolds number, porous Rayleigh number and porous Prandtl number, respectively. Note that the above parameters are related as

$$
R e_{m}=\frac{V_{m}}{V \hat{d}} R e, \quad R a_{m}=\frac{\delta^{2} \epsilon^{2}}{\hat{d}^{4}} R a \quad \text { and } P r_{m}=\epsilon \operatorname{Pr}
$$

where $\delta=\sqrt{K} / d_{m}$ is the Darcy number, $\hat{d}=d / d_{m}$ is the depth ratio and $\epsilon=\alpha / \alpha_{m}$ is the ratio of thermal diffusivity.

The linearized disturbance equations along with the boundary conditions yields a generalized eigen value system of the form

$$
A X=\sigma B X,
$$

where $A$ and $B$ are complex square matrices and $\sigma, X$ are the eigenvalue and eigenvector respectively. The generalized eigenvalue problem is solved by the Chebyshev spectral collocation method [17]. The domain of the Chebyshev polynomials is $[-1,1]$ and in order to reconstruct in the required domain, the field variables in the fluid domain and porous domain are approximated [18] by $\zeta=2 z-1$ and $\zeta_{m}=-2 z_{m}-1$ respectively. The eigenvalues are computed by using QZ algorithm [19] inbuilt in MATLAB software. Also, the accuracy of numerical results is maintained by carrying out various numerical experiments and the maximum order of the chebyshev polynomial hence achieved is 50 . 


\section{Results and Discussion}

The present section probes into the linear stability of plane Poiseuille flow. The analysis focuses on the impact of depth ratio, Darcy number, Reynolds number and Prandtl number. Since, the underlying domain in the system is highly porous, we utilize the physical definition of foametal $\mathrm{C}$ [20] and fix porosity as $\chi=0.78$. As widely used in literature, $\epsilon=0.7$ and $G_{m}=10$ are fixed $[5,6,7]$. For the numerical simulation, three different values of Darcy number: $0.008,0.01$ and 0.05 are considered [21]. Figure $2(a)$ shows the variation of neutral stability curve for different values of depth ratio $(\delta=0.008, \operatorname{Re}=20, \operatorname{Pr}=$ 7 ) with the corresponding oscillatory frequencies shown in figure $2(b)$. It is seen that the neutral curves are bimodal and unimodal depending on the value of depth ratio. The modal characteristic of the neutral curves is defined in the similar means as by Chang [7]. Consequently, porous mode is the one that appears on the left branch with local minimum associated to smaller oscillatory frequency $\sigma_{m}^{r}$ and fluid mode is the one with local minimum associated to larger oscillatory frequency and appearing on right branch. The oscillatory frequencies plotted in figure $2(b)$ serves as the domain of definition and verification for the mode of instability. From figure $2(a)$, it can be seen that for $\hat{d}=0.05$ and 0.1 , the mode of instability is porous and hence unimodal, which is also correspondingly observed from figure $2(b)$, as the oscillatory frequency for $\hat{d}=0.05$ and 0.1 is very much small. As one moves from $\hat{d}=0.1$ to 0.12 , the fluid domain starts to affect the instability and the instability becomes bimodal, where both porous and fluid mode appears. As can be seen from figure 2 , the instability remains bimodal for $\hat{d}=0.12$ to 0.2 , however, on increasing $\hat{d}$ further to 0.5 , the instability again becomes unimodal, but here that single mode is fluid (see the oscillatory frequency pattern for $\hat{d}=0.5$ in figure $2(b)$ ) since the fluid domain completely dominates the instability in this scenario. Physically, as one increases $\hat{d}$, the porous domain's depth simultaneously decreases, which in turn implies an increase in fluid domain's depth, hence, the porous domain receives much less flow for higher values of $\hat{d}$. Therefore, the instability changes from porous to fluid mode with increasing $\hat{d}$. Another observation made from figure 2 is that the critical mode is always the porous mode for $\hat{d} \leq 0.2$.
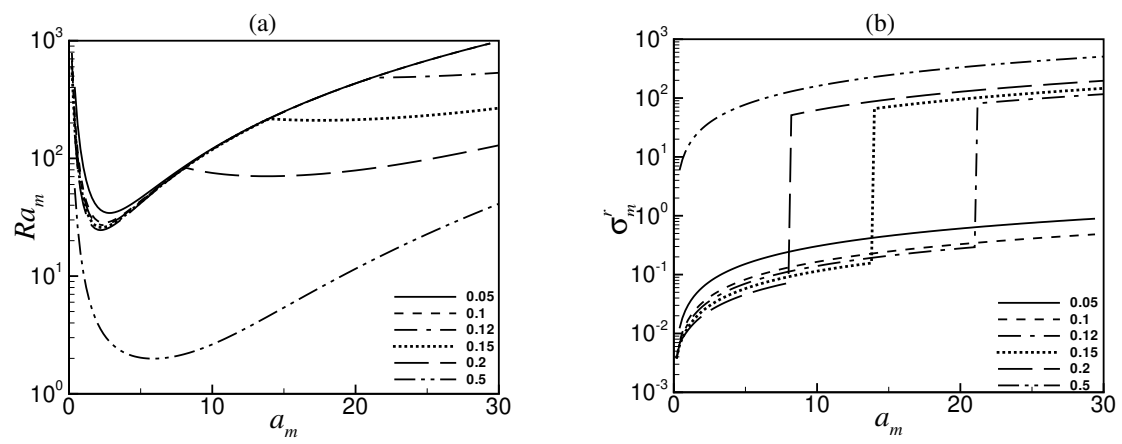

Figure 2: (a) Neutral curves for various values of $\hat{d}$ with $\delta=0.008, \operatorname{Re}=20, \operatorname{Pr}=7$, (b) Corresponding oscillatory frequency variation.

The impact of changing Reynolds number on the stability of the system is shown in figure 3. Figure 3 $(a),(b)$ and $(c)$ presents the neutral curves on variation of Reynolds number for $\delta=0.008,0.01$ and 0.05 respectively with corresponding oscillatory frequencies in $(d)-(f)$. When Darcy number is sufficiently small and $R e \leq 10$, the neutral curve shows bimodal behavior with the porous governing major part of instability for smaller $R e(<20)$. For $R e<20$, as we increase the value of $\delta$ to 0.01 , we observe that the instability due to fluid gradually recedes and due to porous enhances. On further increasing $\delta$ to 0.05 , the instability due to fluid vanishes and the neutral curve exhibit unimodal behavior even for $R e<20$. Furthermore, for $R e \geq 20$, porous mode prevails and the neutral curve is unimodal for all considered values of $\delta$. The critical Rayleigh number increases with increase in $\delta$. It is also witnessed that the critical mode is always porous for any value of $R e$ and $\delta$ under consideration. The above results are legitimate as on increasing the strength of flow i.e. $R e$, fluid easily seeps into the porous domain and instigate the instability there.

Figure 4 exhibits the neutral curves for different values of $\operatorname{Pr}$ and $\delta$. Figure $4(a),(b)$ and $(c)$ presents the variations of Prandtl number for $\delta=0.008,0.01$ and 0.05 respectively with corresponding oscillatory 
frequencies in $(d)-(f)$. From figure $4(a),(b)$, we can see that the neutral curves are bimodal for $\operatorname{Pr}=0.01$ and 0.1 but it changes to unimodal (here, the porous mode) for $\operatorname{Pr} \geq 10$. It can be observed that the fluid domain affects major part of the instability for low $\operatorname{Pr}(0.01) \& \delta=0.008,0.01$ and its influence decreases on increasing $\mathrm{Pr}$. On increasing $\delta$ to 0.01 , the characteristic of the neutral curves remains consistent with those for $\delta=0.008$. However, a further increment in $\delta$ to 0.05 , the neutral curves for all considered values of $\operatorname{Pr}$ become unimodal with porous mode completely dominating the instability in the system. An early achievement of critical Rayleigh number for decreasing $\delta$ is also observed. It is worth noticing that the oscillatory frequency curves for $\operatorname{Pr}=0.01, \delta=0.008$ and $\delta=0.01$ show continuous profiles rather than the usual jumps. Similar pattern for continuous oscillatory profile for low $\operatorname{Pr}$ was observed by Chang [7]. Once again, the least stable mode for any assigned value of $\operatorname{Pr}$ is porous.

(a)

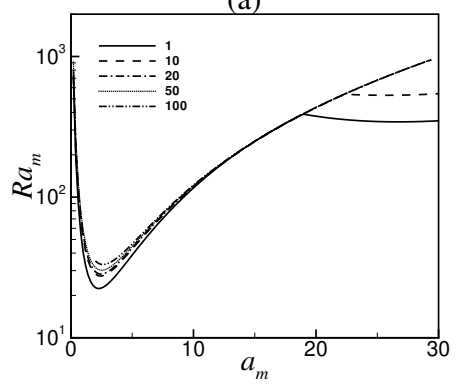

(d)

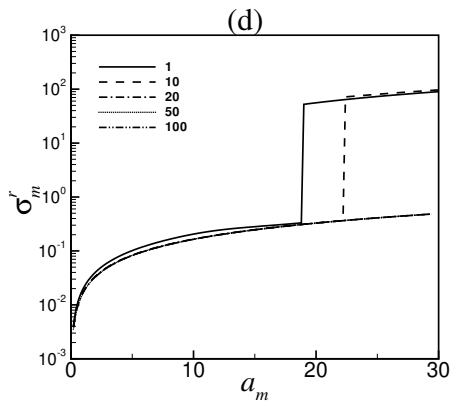

(b)

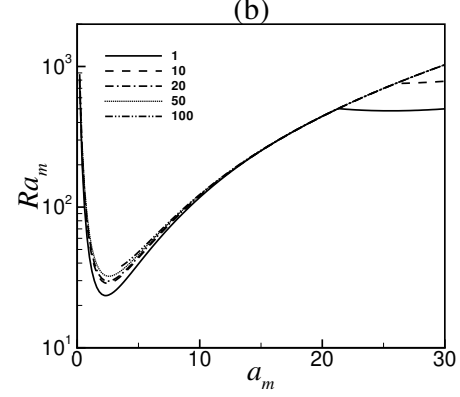

(e)

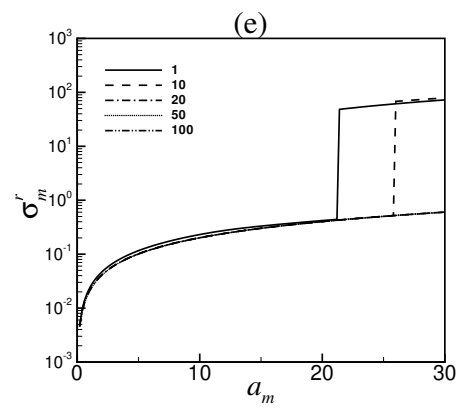

(c)

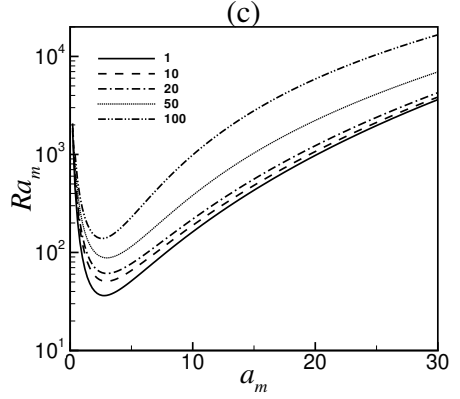

(f)

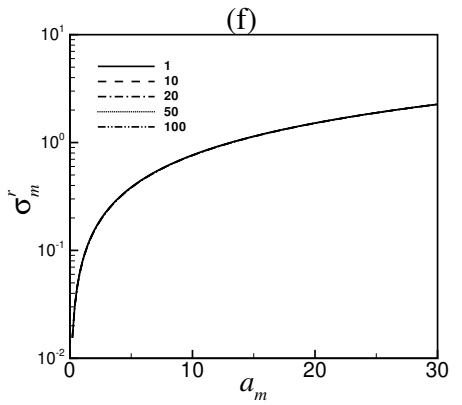

Figure 3: Neutral curves for various values of $\operatorname{Re}$ with $\hat{d}=0.1, \operatorname{Pr}=7$ and $(a) \delta=0.008(b) \delta=0.01$ and $(c) \delta=0.05$ and the corresponding oscillatory frequencies in $(d)-(f)$.

\subsection{Comparison with Darcy's law}

As the two models are different and are utilized in different setups, providing a quantitative comparison becomes challenging. However, qualitative analysis can be employed to provide a meaningful comparison between the models. We have compared our results with the existing results of Chang [7] and our recent work [22] qualitatively. Note that the parameters are different for both studies, as Chang [7] uses $\chi=0.3$ and we adopt $\chi=0.78$. A few qualitative comparisons made are as follow:

- For $0.12 \leq \hat{d} \leq 0.2$, the neutral curves exhibit bimodal nature in both the cases.

- The porous mode instability is achieved much earlier in the Brinkman model w.r.t. considered variation of $R e$.

- The unimodal nature (porous mode) is observed in the Brinkman model for various values of $\operatorname{Pr}$, which was not observed in the results obtained by Darcy's law.

\section{Conclusions}

In the present article, an attempt has been made to study the stability of nonisothermal Poiseuille flow in a fluid overlying a highly porous domain. Brinkman equations are employed to model the porous domain 

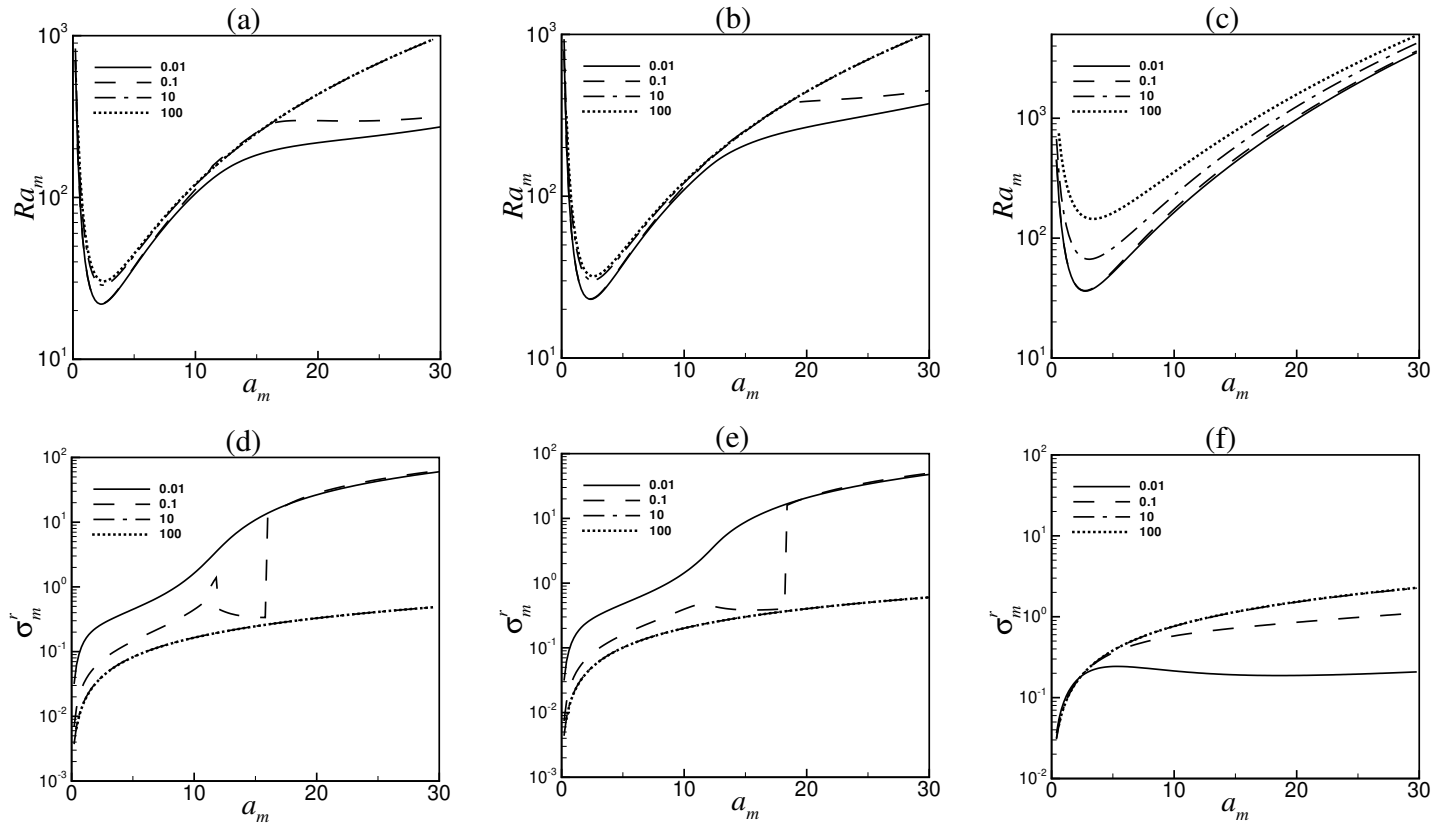

Figure 4: Neutral curves for various values of $\operatorname{Pr}$ with $\hat{d}=0.1, \operatorname{Re}=20$ and $(a) \delta=0.008(b) \delta=0.01$ and $(c) \delta=0.05$ and the corresponding oscillatory frequencies in $(d)-(f)$.

being mindful of the high porosity of the porous region. The results obtained from linear stability analysis assisted in understanding the impact of depth ratio, Darcy number, Reynolds number and Prandtl number on the stability of the superposed system. Increasing the value of depth ratio and decreasing the values of Darcy number, Reynolds number and Prandtl number display change from unimodal (porous) to bimodal. The neutral curve is unimodal with fluid mode only for higher depth ratio viz. $\hat{d}=0.5$. From the numerical analysis, it is viewed that the least stable mode is always the porous mode for all considered values of $\hat{d}, \operatorname{Re}, \operatorname{Pr}$ and $\delta$ except $\hat{d}=0.5$. A careful qualitative comparison with the existing results on Darcy's law is made taking into account the parameters. It is anticipated that the present study will help to understand the appropriate model that should be chosen to model the flow governing one's circumstances. An attempt to study the highly anisotropic and inhomogeneous porous layer below a fluid layer as well as nonlinear stability in such superposed systems can be of great interest. These analyses are kept for our future studies.

\section{Acknowledgment}

The financial support for the present work was partly provided by the Science \& Engineering Research Board, India under the Grant No. SER-1595-MTD, Ministry of Human Resource Development, India and Indian Institute of Technology Roorkee, India.

\section{References}

[1] Discacciati, M., Miglio, E. \& Quarteroni, A.: Mathematical and numerical models for coupling surface and groundwater flows. Applied Numerical Maths, vol. 43: (2002) pp. 57-74.

[2] Allen, M. B.: Collocation Techniques for Modelling Compositional Flows in Oil Reservoirs. Springer, (1984).

[3] Yoshikawa, Y., Akitomo, K. \& Awaji, T.: Formation process of intermediate water in baroclinic current under cooling. Journal of Geophysical Research, vol. 106: (2001) pp. 1033-1051.

[4] Ehrhardt, M., Fuhrmann, J., Holzbecher, E. \& Linke, A.: Mathematical modelling of channel-porous layer interfaces in PEM fuel cells. WIAS, Berlin, (2009) 
[5] Sun W.J.: Convective instability in superposed porous and fluid layers. Ph.D. Dissertation, University of Minnesota, Minneapolis, MN (1973).

[6] Chen, F. \& Chen,C. F.: Onset of finger convection in a horizontal porous layer underlying a fluid layer. Journal of Heat Transfer, vol. 110: (1988) pp. 403-409.

[7] Chang, M. H.: Thermal convection in superposed fluid and porous layers subjected to plane Poiseuille flow. Physics of Fluids vol. 18: (2006) 035104.

[8] Chang, T. Y., Chen, F. \& Chang, M. H.: Stability of plane Poiseuille-Couette flow in a fluid layer overlying a porous layer. Journal of Fluid Mechanics, vol. 826, (2017) pp. 376-395.

[9] Yin, C., Wang, C. \& Wang, S.:Thermal instability of a viscoelastic fluid in a fluid-porous system with a plane Poiseuille flow. Applied Mathematics and Mechanics (English Edition), vol. 41, (2020) pp. 16311650 .

[10] Nield, D. A. \& Bejan, A.: Convection in Porous Media. Springer, New York (2006).

[11] Desaive, Th. \& Lebon, G.: Coupled capillary and gravity-driven instability in a liquid film overlying a porous layer. Physical Review E, vol. 64: (2001) 066304.

[12] Hill, A. A. \& Straughan, B.: Global stability for thermal convection in a fluid overlying a highly porous material. Proceedings of Royal Society A, vol. 465: (2009) pp. 207-217.

[13] Liu, R., Liu, Q. S. \& Zhao, S. C.: Instability of plane Poiseuille flow in a fluid porous system. Physics of Fluids, vol. 20: (2008) 104105.

[14] Hill, A. A. \& Straughan, B.: Poiseuille flow in a fluid overlying a highly porous material. Advances in Water Resources, vol. 32: (2009) pp. 1609-1614.

[15] Ochoa-Tapia, J.A., Whitaker, S.: Momentum transfer at the boundary between a porous medium and a homogeneous fluid i. theoretical development. International Journal of Heat \& Mass Transfer, vol. 38 : (1995) pp. 2635-2646 .

[16] Drazin, P. G. \& Reid, W. H.:Hydrodynamic Stability. Cambridge University Press (2004).

[17] Canuto, C., Hussaini, M. Y., Quarteroni, A. \& Zang, T. A.: Spectral Method in Fluid Dynamics. Springer, New York Berlin Heidelberg (1988).

[18] Khan, A. \& Bera, P.: Linear instability of concentric annular flow: Effect of Prandtl number and gap between cylinders. International Journal of Heat \& Mass Transfer, vol. 15: (2020).

[19] Moler, C.B. \& Stewart, G.W.: An algorithm for generalized matrix eigenvalue problems. SIAM Journal of Numerical Analysis, vol. 20: (1973) pp. 241-256.

[20] Straughan, B.: Effect of property variation and modelling on convection in a fluid overlying a porous layer. International Journal for Numerical and Analytical Methods in Geomechanics, vol. 26:(2002) pp. $75-97$

[21] Samanta, A.: Linear stability of a plane Couette-Poiseuille flow overlying a porous layer. International Journal of Multiphase Flow, vol. 123: (2020) 103160.

[22] Anjali, Khan A., Bera P.: Stability of nonisothermal Poiseuille flow in a fluid overlying an anisotropic and inhomogeneous porous domain. (Submitted:2021) 